\title{
Granulocytic sarcoma of the rectum: a rare complication of myelodysplasia
}

\author{
V Dabbagh, G Browne, L A Parapia, J J Price, P A Batman
}

\section{Department of Histopathology, Bradford Royal Infirmary, Duckworth Lane, Bradford BD9 6RJ, UK \\ V Dabbagh G Browne \\ P A Batman}

\section{Department of Haematology, Bradford Royal Infirmary \\ L A Parapia}

\section{Department of} Surgery, Bradford Royal Infirmary J J Price

Correspondence to: Dr Dabbagh

Accepted for publication 13 July 1999

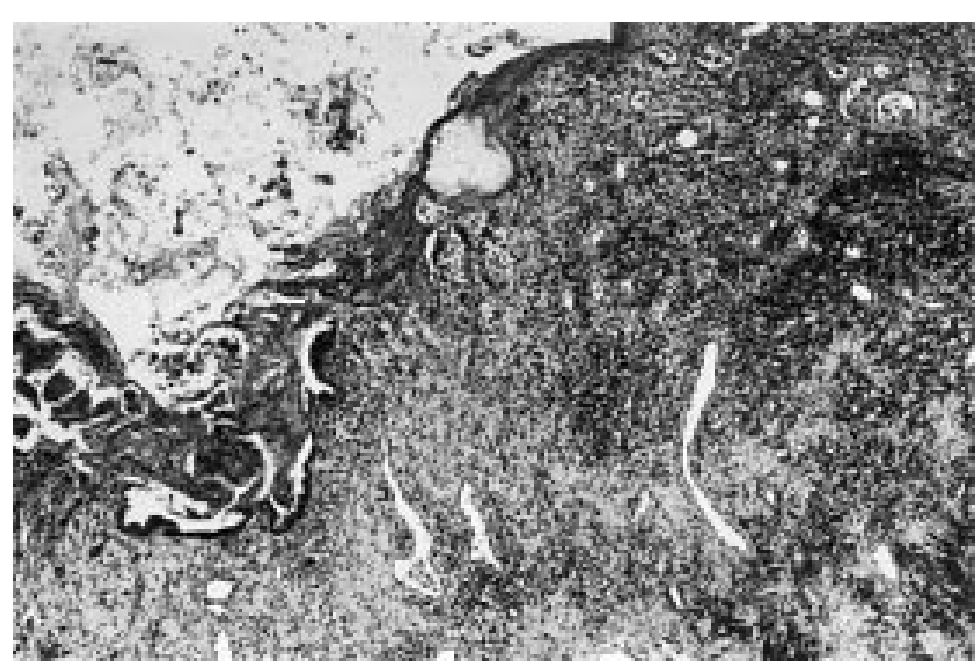

Figure 1 Full thickness mucosal ulceration in the rectum. (Haematoxylin and eosin, magnification $\times 9$.)

Keywords: granulocytic sarcoma; myelodysplasia; rectal cancer

\section{Case report}

A 67 year old man with myelodysplasia requiring blood transfusions was admitted as an emergency with a six week history of rectal bleeding and diarrhoea. Eleven months earlier a bone marrow trephine biopsy showed hypercellular marrow with marked erythroid hyperplasia with megaloblastic and dysplastic changes. Granulopoiesis was relatively decreased with no increase in blasts. No ring sideroblasts were present.

A full blood count showed a haemoglobin of $7.5 \mathrm{~g} / \mathrm{dl}$, white cell count of $1.0 \times 10^{9} / 1$ and a platelet count of $18 \times 10^{9} / 1$. The blood film failed to show any blasts. Sigmoidoscopy revealed an inflamed mucosa with contact bleeding, and a barium enema showed an irregular polypoid filling defect in the lateral

\begin{abstract}
A 67 year old man with myelodysplasia was admitted as an emergency with a six week history of rectal bleeding and diarrhoea. Barium enema showed an irregular polypoid filling defect in the lateral wall of the proximal rectum near the rectosigmoid junction. Histology showed this to be a granulocytic sarcoma (extramedullary granulocytic leukaemia; chloroma) infiltrating the bowel. A low index of suspicion of this lesion results in an incorrect diagnosis in many such cases. A chloroacetate esterase immunoperoxidase stain will confirm the granulocytic nature of the tumour cells.

(f Clin Pathol 1999;52:865-866)
\end{abstract}

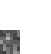

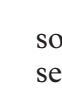
medium sized arteries and veins in the serosal tissue, with focal fibrosis of the muscularis propria and haemosiderin deposition in the submucosa. This pathology implies a degree of ischaemia in the aetiology of the ulcer, possibly produced by tumour involvement of vessels.

\section{Discussion}

Granulocytic sarcoma is an extramedullary tumour composed of immature cells of the granulocytic series (myeloid cells). It was first described by Burns in 1811, and because such tumours display a greenish colour owing to the presence of myeloperoxidase in the cells, the term chloroma was coined in 1853 by King. ${ }^{2}$ This tumour mass may arise in four clinicopathological situations: (1) a harbinger of acute myeloid leukaemia in a non-leukaemic patient; 


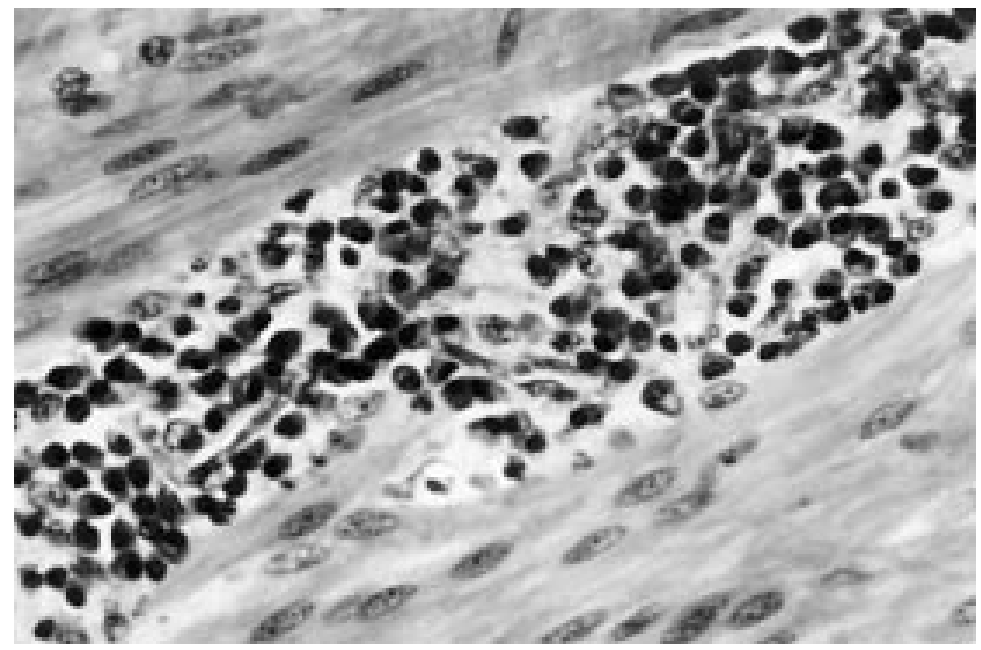

Figure 2 Dense infiltrate of monotonous medium sized nuclei with inconspicuous nucleoli. (Haematoxylin and eosin, magnification $\times 36$.)

(2) impending blast crisis in chronic myeloid leukaemia or leukaemic transformation in myelodysplastic disorders; (3) an additional complication of known acute leukaemia; and (4) an isolated lesion. ${ }^{3}$

The most common presentation is a tumour confined to a single site, although one third of patients with chronic myeloid leukaemia are found to have foci of extramedullary leukaemia at necropsy. ${ }^{4}$ Granulocytic sarcoma may occur in virtually any organ, although the central nervous system, bones, ovaries, and lymph nodes are most frequently involved. Tumours of the small or large intestine have seldom been described; this involvement is usually asymptomatic, but patients may present with acute abdominal pain or change in bowel habit with rectal bleeding. ${ }^{356}$

Granulocytic sarcoma may range from well differentiated tumours on histological examination to those with virtually no evidence of myeloid differentiation. A low index of suspicion of the lesion results in an incorrect diagnosis in many cases ${ }^{7}$; however, the chloroacetate esterase immunoperoxidase stain will confirm the granulocytic nature of the tumour cells. The lesion in the present case presented both radiologically and macroscopically as a common rectal carcinoma; consideration of the alternative correct diagnosis of granulocytic sarcoma in the appropriate clinical context is important, since chemotherapy or radiotherapy are then treatment options. ${ }^{3}$

1 Burns A. Observations of surgical anatomy in head and neck. Edinburgh: Thomas Royce, 1811:364-6.

2 King A. A case of chloroma. Monthly f Med 1853;17:97.

3 Catalano MF, Levin B, Hart RS, et al. Granulocytic sarcoma of the colon. Gastroenterology 1991;100:555-9.

4 Rosenthal S, Cannellos G, De Vita VT, et al. Characteristics of blast crisis in chronic granulocytic leukaemia. Blood 1977;49:705-19.

5 Lugassy G, Gimon Z, Okon E, et al. Acute abdomen due to granulocytic sarcoma of the terminal ileum. Eur f Surg Oncol 1988;14:445-8.

6 Rottenbery GT, Thomas BM. Case report: granulocytic sarcoma of the small bowel - a rare presentation of leukaemia. Clin Radiol 1994;49:501-2.

7 Meiss MJ, Butler JJ, Osborne BM, et al. Granulocytic sarcoma in nonleukaemic patients. Cancer 1986;58:2697709. 